\title{
OPEN Genetically predicted education attainment in relation to somatic and mental health
}

\author{
Shuai Yuan ${ }^{1}$, Ying Xiong ${ }^{2}$, Madeleine Michaëlsson ${ }^{3}$, Karl Michaëlsson $^{4}$ \& \\ Susanna C. Larsson ${ }^{1,4 \bowtie}$
}

A deeper understanding of the causal links from education level to health outcomes may shed a light for disease prevention. In the present Mendelian randomization study, we found that genetically higher education level was associated with lower risk of major mental disorders and most somatic diseases, independent of intelligence. Higher education level adjusted for intelligence was associated with lower risk of suicide attempts, insomnia, major depressive disorder, heart failure, stroke, coronary artery disease, lung cancer, breast cancer, type 2 diabetes and rheumatoid arthritis but with higher risk of obsessive-compulsive disorder, anorexia nervosa, anxiety, bipolar disorder and prostate cancer. Higher education level was associated with reduced obesity and smoking, which mediated quite an extent of the associations between education level and health outcomes. These findings emphasize the importance of education to reduce the burden of common diseases.

Education level is an important health social determinant and has been proposed as a modifiable risk factor for a number of disorders and diseases, such as depression ${ }^{1}$, age-related cognitive decline ${ }^{2}$, suicide ${ }^{3}$, cardiovascular disease $^{4}$, cancer $^{5}$, and several other diseases ${ }^{6-8}$. However, it is unclear whether the associations are causal and independent of intelligence. Understanding the causal effects of education level on diseases can facilitate the aetiology pathway exploration of diseases as well as development of new strategies for disease prevention. Notwithstanding, randomized controlled trials are ethically and practically infeasible on this topic.

Exploiting genetic variants as instrumental variables for an exposure (i.e., education level), Mendelian randomization (MR) can strengthen the causal inference of an exposure-outcome association ${ }^{9}$. Comparing the risk of disease across individuals who have been classified by their genotype enables the causal effect of an exposure to be estimated with substantially less bias, such as confounding and reverse causality, than in a traditional observational analysis ${ }^{9}$. The rationale for diminished bias in MR studies is that genetic variants are randomly assorted and fixed at conception and therefore largely independent of confounders and cannot be modified by disease development ${ }^{9}$.

Several previous MR studies revealed possible causal associations of genetically higher education level with health outcomes, such as Alzheimer's disease ${ }^{10,11}$, diabetes ${ }^{12}$, cardiovascular disease ${ }^{13-15}$, cancer ${ }^{16}$, myopia $^{8}$, chronic kidney disease ${ }^{17}$, amyotrophic lateral sclerosis ${ }^{18}$, and longevity ${ }^{19}$. In a Mendelian randomization study based on UK Biobank, however, the pattern of the protective effect of higher genetically predicted education level on a broad range of health endpoints was unclear ${ }^{20}$. Education is an upstream health determinant that influences social and community networks and individual lifestyle factors, thereby affecting the risk of various health outcomes. Higher educational attainment has been established to have direct effects on income, alcohol consumption, and physical activity, and inverse associations with smoking, BMI and sedentary behavior. Although genetically proxied higher education level has been associated with several disease ${ }^{20}$, whether education exerts causal effects on a wide spectrum of health outcomes remains unknown.

Here, we conducted an MR study to disentangle the causal role of education level from intelligence in major mental and neurological disorders and somatic diseases. A secondary aim was to explore whether intelligence is causally associated with the same health outcomes independently of education. We additionally investigated the associations of education level and intelligence with modifiable health-related risk factors. Given that obesity and

\footnotetext{
${ }^{1}$ Unit of Cardiovascular and Nutritional Epidemiology, Institute of Environmental Medicine, Karolinska Institutet, Nobelsväg 13, 17177 Stockholm, Sweden. ${ }^{2}$ Department of Public Health Sciences, Karolinska Institutet, Stockholm, Sweden. ${ }^{3}$ Department of Education, Health and Social Studies, Dalarna University, Falun, Sweden. ${ }^{4}$ Unit of Medical Epidemiology, Department of Surgical Sciences, Uppsala University, Dag Hammarskjölds Väg 14B, 75185 Uppsala, Sweden. ${ }^{凶}$ email: susanna.larsson@surgsci.uu.se
} 
smoking influence the risk of many diseases ${ }^{21-25}$, we examined whether these two factors mediate the pathway from education to health outcomes.

\section{Materials and methods}

Study design. The design and hypothesis of the present study are displayed in Supplementary Fig. 1. We used summary-level data from large genome-wide association studies (GWASs) and genetic consortia (Table 1). Totally, our study included 11 mental and neurological disorders, 19 major somatic diseases, body mass index and cigarette smoking. A systematic review was conducted to find meta-analyses of observational studies of education level and diseases (Supplementary Table 1). All GWASs had been approved by a relevant ethical review board and participants had given informed consent. No individual-level data were used in the present MR study. This MR study was approved by the Swedish Ethical Review Authority.

Selection of instrumental variables. Instrumental variables for education level and intelligence were identified from GWASs of, respectively, 1,131,881 and 269,867 individuals of European ancestries ${ }^{26,27}$. In total, 1271 and 205 single-nucleotide polymorphisms (SNPs) at the genome-wide significance threshold $\left(p<5 \times 10^{-8}\right)$ were identified to be associated with education level and intelligence, respectively. Independent SNPs $\left(r^{2}<0.01\right.$ and clump window $>10 \mathrm{~kb}$ ) without linkage disequilibrium were proposed as instrumental variables. Linkage disequilibrium among SNPs was calculated based on 1000 genomes LD reference panel (European population) using the PLINK clumping method. Possible palindromic SNPs were excluded. We used 663 and 178 SNPs as instrumental variables for education level and intelligence, respectively. The same instrumental variables were used in the multivariable MR analyses. Education level was defined as number of years of education and was unified across included studies according to an International Standard Classification of Education category. The sample-size-weighted mean of education year was 16.8 years of schooling with a standard deviation (SD) of 4.2 years. For the definition of intelligence, included cohorts extracted a single sum score, mean score, or factor score from a multidimensional set of cognitive performance tests in GWAS with linear model, with the exception of High-IQ/Health and Retirement Study where a logistic regression GWAS was run with "case" status (high intelligence) versus controls (normal intelligence level). All included GWASs adjusted for key covariates, such as age, sex and principal components for ancestry.

Outcome sources. Summary-level data for the associations of the education- and intelligence-associated SNPs with the outcomes were extracted from large-scale GWASs or genetic consortia. In the present MR study, we included 11 mental and neurological disorders ${ }^{28-38}, 9$ cardiovascular diseases $^{39-43}, 3$ major cancers ${ }^{44-46}, 7$ other diseases ${ }^{47-53}$, body mass index ${ }^{54}$ and cigarette smoking ${ }^{55}$. We did not find proxies for exposure-associated SNPs that were unavailable in the outcome datasets given that the percentage of missing SNPs for most outcomes were minimal and a few missing SNPs was not likely to bias the results based on hundreds of SNPs. Detailed information, such as the number cases and controls, population structure and the source for each outcome, is presented in Table 1. Definitions of the outcomes are presented in Supplementary Table 2.

Systematic review for meta-analysis of observational studies. A systematic literature search was conducted in the PubMed database before November 1st, 2019 to find meta-analyses of observational studies of education level in relation to diseases studied in the present MR study. We found latest published meta-analysis on 13 diseases and two risk factors, including major depressive disorders ${ }^{56}$, suicide attempts ${ }^{57}$, posttraumatic stress disorder ${ }^{58}$, amyotrophic lateral sclerosis ${ }^{59}$, Alzheimer's disease ${ }^{60}$, coronary artery disease ${ }^{61}$, heart failure , $^{62}$, stroke $^{63}$, breast cancer ${ }^{64}$, prostate cancer ${ }^{65}$, lung cancer ${ }^{66}$, type 2 diabetes $^{67}$, chronic kidney disease ${ }^{68}$ and body mass index ${ }^{69}$. We extracted publication data (PubMed identifier number, the first author's name and year of publication), sample size, and risk estimates with their corresponding confidence intervals. Search strategy and characteristics of included meta-analyses are shown in Supplementary Table 1.

Statistical analysis. The random-effects inverse-variance weighted method was used to assess the associations of education and intelligence with the outcomes. The weighted median method and MR-Egger regression were used as sensitivity analyses to examine the consistency of results and to detect potential pleiotropy. The weighted median method gives accurate estimates if at least $50 \%$ of the instrumental variables are valid ${ }^{70}$. The MR-Egger regression can detect and adjust for pleiotropy albeit rendering low precision of the estimates ${ }^{71}$. The false discovery rate method was used to adjust for multiple testing (Supplementary Tables 3 and 4 ). For associations that survived multiple testing, we used the multivariable MR method ${ }^{72}$ to disentangle the causal effect of education level on outcomes independent of intelligence and vice versa. For intelligence-adjusted inverse associations that survived multiple testing, we also used the multivariable MR analysis with adjustment for body mass index and smoking to explore the mediation effects of these factors on the associations between education and health outcomes. We performed several multivariable MR analyses to test the mediation effect from education, intelligence, body mass index or smoking, rather than allowing for independent effects as well as mediations by these factors in one MVMR model simultaneously.

Proportions of attenuated effect size were calculated to present the magnitude of mediation effects. Odds ratios (ORs) and 95\% confidence intervals (CIs) of diseases and changes of levels of risk factors were scaled to an SD increase in genetically predicted years of education (4.2 years) and intelligence. All statistical analyses were two-sided and performed using the mrrobust package ${ }^{73}$ in Stata/SE 15.0 (StataCorp. 2017. Stata Statistical Software: Release 15. College Station, TX: StataCorp LLC.) and TwoSampleMR ${ }^{74}$ in R Software 3.6.0 (R Core Team. R Foundation for Statistical Computing. Vienna, Austria. 2019. https://www.R-project.org). 


\begin{tabular}{|c|c|c|c|c|c|}
\hline Outcome & Cases, No & Controls, No & Population & Study source & Data source \\
\hline \multicolumn{6}{|l|}{ Mental disorder } \\
\hline Anorexia nervosa & 16,992 & 55,525 & European & Watson HJ et al. & $\begin{array}{l}\text { Psychiatric Genomics Con- } \\
\text { sortium }\end{array}$ \\
\hline Anxiety & 7016 & 14,745 & European & Otowa $\mathrm{T}$ et al. & $\begin{array}{l}\text { Psychiatric Genomics Con- } \\
\text { sortium }\end{array}$ \\
\hline Bipolar disorder & 20,352 & 31,358 & European & Stahl EA et al. & $\begin{array}{l}\text { Psychiatric Genomics Con- } \\
\text { sortium }\end{array}$ \\
\hline Insomnia & 397,959 & 933,051 & European & Jansen PR et al. & CNCR \\
\hline Major depressive disorder & 170,756 & 329,443 & European & Stahl EA et al. & UK Biobank \\
\hline $\begin{array}{l}\text { Obsessive-compulsive } \\
\text { disorder }\end{array}$ & 2688 & 7037 & European & IOCDF-GC and OCGAS & $\begin{array}{l}\text { Psychiatric Genomics Con- } \\
\text { sortium }\end{array}$ \\
\hline Posttraumatic stress disorder & 30,000 & 170,000 & Mix & Nievergelt CM et al. & $\begin{array}{l}\text { Psychiatric Genomics Con- } \\
\text { sortium }\end{array}$ \\
\hline Suicide attempts & 6024 & 44,240 & European & Erlangsen A et al. & iPSYCH \\
\hline Schizophrenia & 33,426 & 54,065 & European & $\begin{array}{l}\text { Psychiatric Genomics Con- } \\
\text { sortium }\end{array}$ & $\begin{array}{l}\text { Psychiatric Genomics Con- } \\
\text { sortium }\end{array}$ \\
\hline \multicolumn{6}{|l|}{ Neurological disease } \\
\hline Amyotrophic lateral sclerosis & 21,982 & 41,944 & European & Kunkle BW et al. & Project MinE \\
\hline Alzheimer's disease & 12,577 & 23,475 & European & van Rheenen W et al. & IGAP \\
\hline \multicolumn{6}{|l|}{ Cardiovascular disease } \\
\hline Atrial fibrillation & 65,446 & 522,000 & Mix & Roselli C et al. & AFGen \\
\hline Coronary artery disease & 60,801 & 123,504 & Mix & Nikpay M et al. & $\begin{array}{l}\text { CARDIoGRAMplusC4D } \\
\text { Consortium }\end{array}$ \\
\hline Heart failure & 7382 & 387,652 & European & Aragam KG et al. & UK Biobank \\
\hline Total stroke & 67,162 & 454,450 & Mix & Malik R et al. & MEGASTROKE Consortium \\
\hline Any ischemic stroke & 60,341 & NA & Mix & Malik R et al. & MEGASTROKE Consortium \\
\hline Large artery stroke & 6688 & 146,392 & Mix & Malik R et al. & MEGASTROKE Consortium \\
\hline Small vessel stroke & 11,710 & 192,662 & Mix & Malik R et al. & MEGASTROKE Consortium \\
\hline Cardioembolic stroke & 9006 & 204,570 & Mix & Malik R et al. & MEGASTROKE Consortium \\
\hline Intracerebral haemorrhage & 1545 & 1481 & Mix & Woo D et al. & ISGC \\
\hline \multicolumn{6}{|l|}{ Cancer } \\
\hline Breast cancer & 122,977 & 105,974 & Mix & Michailidou K et al. & BCAC \\
\hline Breast cancer ER+ & 69,501 & NA & Mix & Michailidou K et al. & BCAC \\
\hline Breast cancer ER- & 21,468 & NA & Mix & Michailidou K et al. & BCAC \\
\hline Lung cancer & 11,348 & 15,861 & European & Wang Y et al. & ILCCO \\
\hline Prostate cancer & 79,194 & 61,112 & European & Schumacher FR et al. & PRACTICAL Consortium \\
\hline \multicolumn{6}{|l|}{ Other disease } \\
\hline Atopic dermatitis & 21,399 & 95,464 & Mix & Paternoster L et al. & EAGLE Consortium \\
\hline Chronic kidney disease & 41,395 & 439,303 & European & Wuttke $\mathrm{M}$ et al. & CKDGen Consortium \\
\hline Fracture & 53,184 & 373,611 & European & Morris JA et al. & GEFOS Consortium \\
\hline Gout & 13,179 & 750,634 & Mix & Tin A et al. & GUGC \\
\hline Inflammatory bowel disease & 25,042 & 34,915 & European & de Lange KM et al. & UK IBD consortium \\
\hline Rheumatoid arthritis & 29,880 & 73,758 & Mix & Okada Y et al. & GARNET consortium \\
\hline Type 2 diabetes & 74,124 & 824,006 & European & Mahajan A et al. & DIAGRAM consortium \\
\hline \multicolumn{6}{|l|}{ Risk factor } \\
\hline Body mass index & NA & 694,649 & Mix & Pulit SL et al. & GIANT consortium \\
\hline Cigarettes per day & NA & 337,334 & European & Liu $\mathrm{M}$ et al. & GSCAN \\
\hline
\end{tabular}

Table 1. Characteristics of included studies of mental disorders, somatic diseases, and health-related risk factors. AFGen Atrial Fibrillation Consortium, BCAC Breast Cancer Association Consortium, CNCR Center for Neurogenomics and Cognitive Research, DIAGRAM The DIAbetes Genetics Replication And Metaanalysis, EAGLE The EArly Genetics and Lifecourse Epidemiology, ER estrogen receptor, GARNET Genetics and Allied research in Rheumatic diseases Networking, GEFOS GEnetic Factors for Osteoporosis, GUGC The Global Urate Genetics Consortium, GSCAN Consortium of Alcohol and Nicotine use, IGAP The International Genomics of Alzheimer's Project, ILCCO The International Lung Cancer Consortium, ISGC International Stroke Genetics Consortium, NA Not available, PRACTICAL The Prostate Cancer Association Group to Investigate Cancer Associated Alterations in the Genome, SNP single-nucleotide polymorphism, UK IBD consortium UK Inflammatory Bowel Disease Genetics Consortium. 


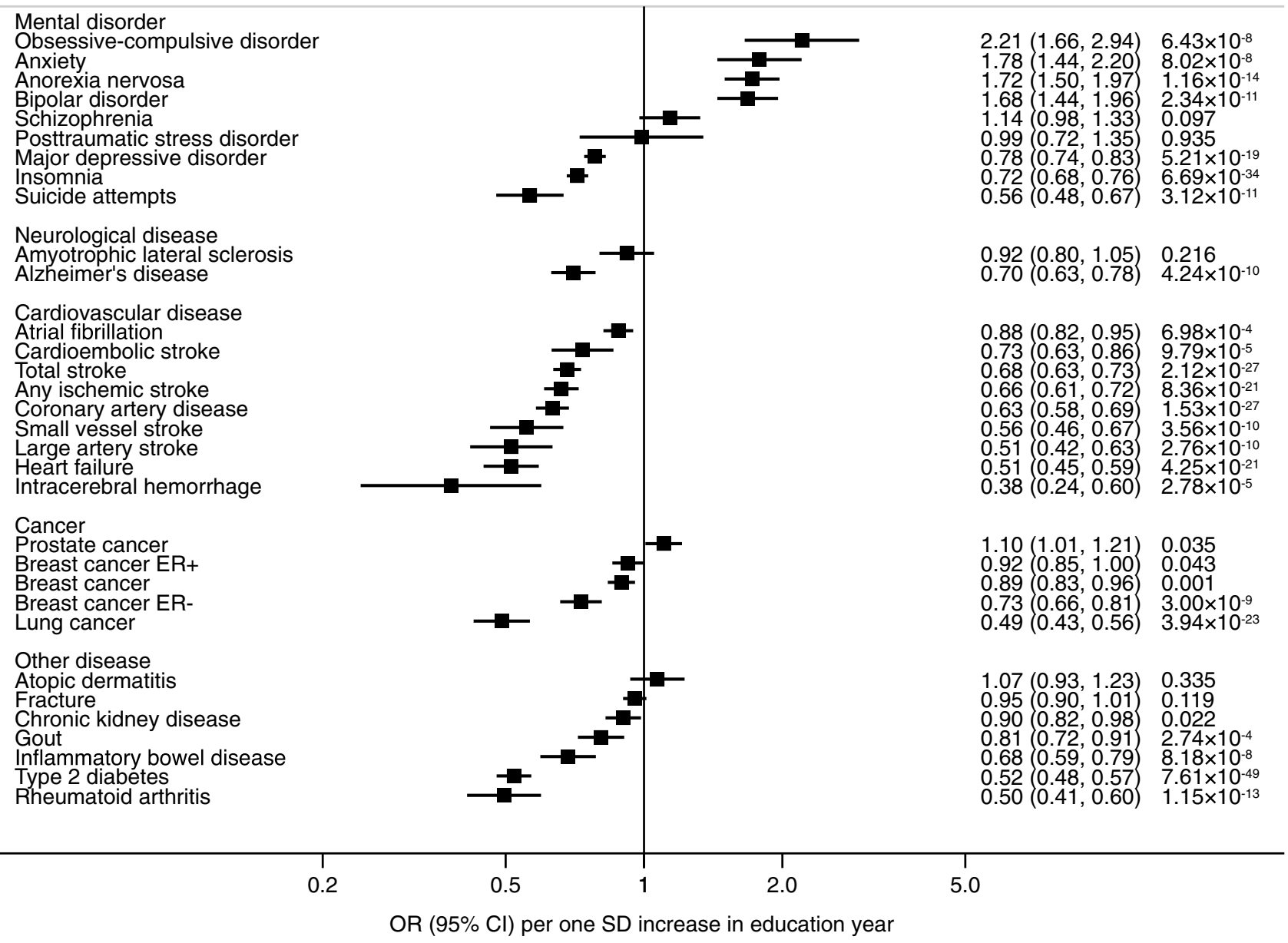

Figure 1. Associations of genetic predisposition to higher education level with health outcomes in univariable $\mathrm{MR}$ analyses. $C I$ confidence interval, $E R$ oestrogen receptor, $I V W$ inverse-variance weighted, $O R$ odds ratio, $S D$ standard deviation.

Ethical approval. This MR study was approved by the Swedish Ethical Review Authority.

Informed consent. All participants included in the genome-wide association studies gave informed consent.

\section{Results}

Genetically predicted education level and diseases. Genetically predicted education level was causally associated with most diseases, including 8 out of 11 mental and neurological disorders, all 9 studied cardiovascular diseases, all 3 studied cancers, and 5 out of 7 other common diseases in the univariable inverse-variance weighted MR analysis (Fig. 1 and Supplementary Table 3). In the multivariable inverse-variance weighted analysis, the associations of education level with Alzheimer's disease, atrial fibrillation, cardioembolic stroke, intracerebral haemorrhage, chronic kidney disease, gout, and inflammatory bowel disease did not remain after adjustment for intelligence (Fig. 2 and Supplementary Table 3). Results of sensitivity analyses were directionally similar but with wider CIs (Supplementary Table 5).

Genetically predicted intelligence and diseases. The associations between intelligence and outcomes are presented in Supplementary Tables 4,6 and 7. Genetically predicted intelligence showed associations with obsessive-compulsive disorder, anorexia nervosa, schizophrenia, insomnia, suicide attempts, Alzheimer's disease, coronary artery disease, breast cancer (ER-), lung cancer, type 2 diabetes and rheumatoid arthritis in the univariable MR analyses (Supplementary Tables 4 and 6). After adjustment for genetically predicted education level, only the association with schizophrenia persisted (Supplementary Tables 4 and 7).

Education, intelligence, body mass index and smoking. Genetically predicted higher education level was associated with lower body mass index and fewer cigarettes smoked per day in the univariable model; the estimates were similar in the intelligence-adjusted model (Fig. 3 and Supplementary Tables 3 and 8). Find- 
Mental disorder

Obsessive-compulsive disorder

Bipolar disorder

Anxiety

Anorexia nervosa

Major depressive disorder

Insomnia

Suicide attempts

Neurological disease Alzheimer's disease

Cardiovascular disease Atrial fibrillation

Cardioembolic stroke

Coronary artery disease

Total stroke

Any ischemic stroke

Small vessel stroke

Large artery stroke

Heart failure

Intracerebral hemorrhage

Cancer

Prostate cancer

Breast cancer

Breast cancer ER-

Lung cancer

Other disease

Chronic kidney disease

Gout

Inflammatory bowel disease

Type 2 diabetes

Rheumatoid arthritis

\section{der

(1)

2.35 (1.36, 4.04) 0.002

$1.87(1.40,2.49) \quad 2.05 \times 10^{-5}$

$1.79(1.18,2.71) \quad 0.006$

$1.61(1.24,2.09) \quad 3.44 \times 10^{-4}$

$0.73(0.66,0.81) \quad 3.60 \times 10^{-9}$

$0.70(0.63,0.77) \quad 9.62 \times 10^{-12}$

$0.43(0.31,0.59) \quad 1.83 \times 10^{-7}$

$0.92(0.74,1.13) \quad 0.426$
$0.88(0.77,1.02) \quad 0.087$

$0.81(0.60,1.08) \quad 0.153$

$0.77(0.66,0.89) \quad 0.001$

$0.72(0.63,0.82) \quad 6.30 \times 10^{-7}$

$0.67(0.57,0.79) \quad 1.65 \times 10^{-6}$

$0.51(0.36,0.72) \quad 1.29 \times 10^{-4}$

$0.50(0.34,0.74) \quad 0.001$

$0.48(0.37,0.63) \quad 5.07 \times 10^{-8}$

$0.44(0.18,1.06) \quad 0.066$

$1.22(1.02,1.44) \quad 0.028$

$0.85(0.75,0.97) \quad 0.014$

$0.72(0.59,0.87) \quad 0.001$

$0.54(0.41,0.70) \quad 4.84 \times 10^{-6}$

$0.91(0.77,1.08) \quad 0.284$

$0.84(0.67,1.04) \quad 0.112$

$0.81(0.62,1.05) \quad 0.108$

$0.50(0.42,0.59) \quad 7.42 \times 10^{-17}$

$0.49(0.34,0.69) \quad 6.82 \times 10^{-5}$

$\begin{array}{lllll}1 & 1 & 1 & 1 \\ 0.2 & 0.5 & 1 & 2.0 & 5.0\end{array}$

OR $(95 \% \mathrm{Cl})$ per one SD increase in education year

Figure 2. Associations of genetic predisposition to higher education level with health outcomes in multivariable MR analyses with adjustment for genetically predicted intelligence. CI confidence interval, ER oestrogen receptor, $I V W$ inverse-variance weighted, $O R$ odds ratio, $S D$ standard deviation.

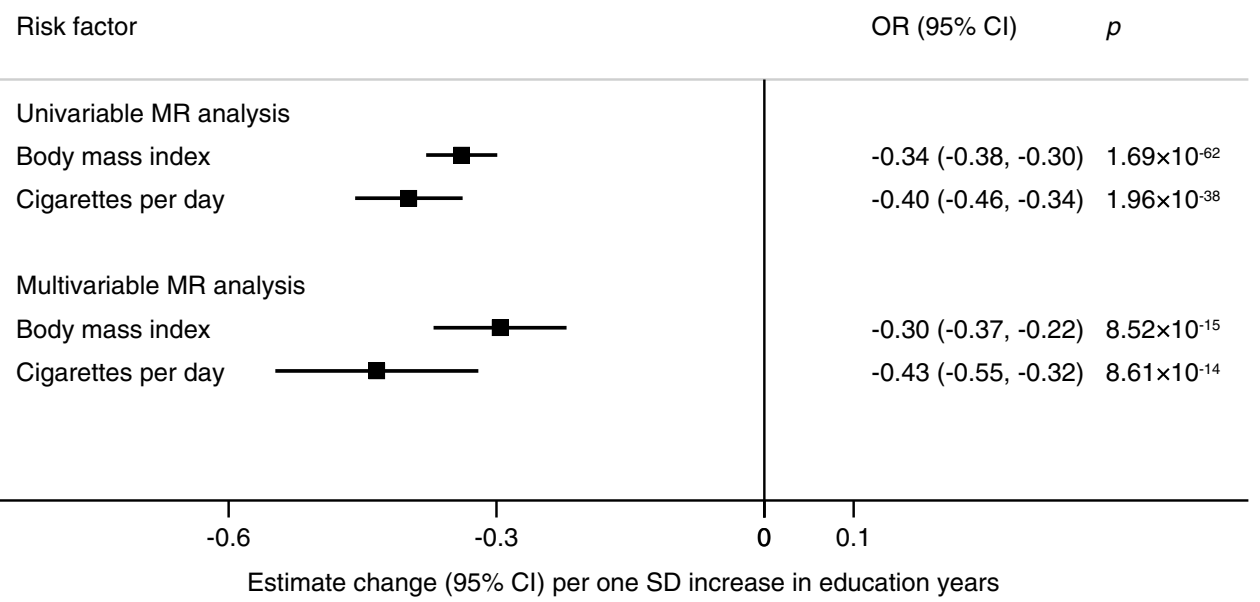

Figure 3. Associations of genetic predisposition to higher education level with body mass index and smoking in MR analyses without and with adjustment for genetically predicted intelligence. CI confidence interval, $I V W$ inverse-variance weighted, $S D$ standard deviation. 


\begin{tabular}{|c|c|c|c|c|c|c|c|c|c|c|c|}
\hline \multirow[b]{2}{*}{ Outcomes } & \multicolumn{2}{|c|}{$\begin{array}{l}\text { Total effect of } \\
\text { education }\end{array}$} & \multicolumn{3}{|c|}{$\begin{array}{l}\text { Effect after adjusting } \\
\text { for BMI }\end{array}$} & \multicolumn{3}{|c|}{$\begin{array}{l}\text { Effect after adjusting } \\
\text { for smoking }\end{array}$} & \multicolumn{3}{|c|}{$\begin{array}{l}\text { Effect after adjusting } \\
\text { for both }\end{array}$} \\
\hline & OR $^{\mathbf{a}}$ & $95 \% \mathrm{CI}$ & $\mathbf{O R}^{\mathbf{b}}$ & $95 \% \mathrm{CI}$ & $\%^{*}$ & $\mathrm{OR}^{\mathrm{c}}$ & $95 \% \mathrm{CI}$ & $\%^{*}$ & OR $^{\mathrm{d}}$ & $95 \% \mathrm{CI}$ & $\%^{*}$ \\
\hline \multicolumn{12}{|l|}{ Mental disorder } \\
\hline Insomnia & 0.72 & $0.68,0.76$ & 0.75 & $0.71,0.80$ & 12 & 0.74 & $0.69,0.79$ & 8 & 0.77 & $0.72,0.82$ & 20 \\
\hline Major depressive disorder & 0.78 & $0.74,0.83$ & 0.85 & $0.80,0.90$ & 35 & 0.83 & $0.77,0.88$ & 25 & 0.87 & $0.81,0.93$ & 44 \\
\hline Suicide attempts & 0.56 & $0.48,0.67$ & 0.60 & $0.50,0.72$ & 12 & 0.67 & $0.54,0.82$ & 31 & 0.68 & $0.55,0.84$ & 33 \\
\hline \multicolumn{12}{|l|}{ Cardiovascular disease } \\
\hline Coronary artery disease & 0.63 & $0.58,0.69$ & 0.67 & $0.61,0.73$ & 13 & 0.72 & $0.65,0.79$ & 29 & 0.74 & $0.66,0.82$ & 35 \\
\hline Heart failure & 0.51 & $0.45,0.59$ & 0.55 & $0.47,0.64$ & 11 & 0.64 & $0.54,0.75$ & 34 & 0.65 & $0.55,0.77$ & 36 \\
\hline Total stroke & 0.68 & $0.63,0.73$ & 0.70 & $0.64,0.75$ & 8 & 0.75 & $0.69,0.81$ & 25 & 0.76 & $0.69,0.82$ & 29 \\
\hline Any ischemic stroke & 0.66 & $0.61,0.72$ & 0.67 & $0.61,0.74$ & 4 & 0.69 & $0.63,0.77$ & 11 & 0.70 & $0.63,0.78$ & 14 \\
\hline Large artery stroke & 0.51 & $0.42,0.63$ & 0.54 & $0.43,0.68$ & 8 & 0.59 & $0.46,0.76$ & 22 & 0.59 & $0.46,0.77$ & 22 \\
\hline Small vessel stroke & 0.56 & $0.46,0.67$ & 0.54 & $0.44,0.67$ & 0 & 0.54 & $0.43,0.67$ & 0 & 0.53 & $0.42,0.66$ & 0 \\
\hline \multicolumn{12}{|l|}{ Cancer } \\
\hline Breast cancer & 0.89 & $0.83,0.96$ & 0.92 & $0.85,1.00$ & 28 & 0.93 & $0.85,1.01$ & 38 & 0.94 & $0.86,1.03$ & 47 \\
\hline Breast cancer ER- & 0.73 & $0.66,0.81$ & 0.74 & $0.66,0.83$ & 4 & 0.71 & $0.63,0.80$ & 0 & 0.71 & $0.63,0.81$ & 0 \\
\hline Lung cancer & 0.49 & $0.43,0.56$ & 0.56 & $0.48,0.65$ & 19 & 0.53 & $0.45,0.63$ & 11 & 0.59 & $0.49,0.70$ & 26 \\
\hline \multicolumn{12}{|l|}{ Other diseases } \\
\hline Rheumatoid arthritis & 0.50 & $0.41,0.60$ & 0.44 & $0.36,0.53$ & 0 & 0.41 & $0.34,0.50$ & 0 & 0.42 & $0.34,0.52$ & 0 \\
\hline Type 2 diabetes & 0.52 & $0.48,0.57$ & 0.60 & $0.55,0.64$ & 22 & 0.75 & $0.70,0.81$ & 56 & 0.79 & $0.73,0.85$ & 64 \\
\hline
\end{tabular}

Table 2. Mediation analysis to disentangle the effects of body mass index and smoking in the pathway from education level to health outcomes. BMI body mass index, ER estrogen receptor. ${ }^{\star}$ Percentage of the effect of education on the health outcome that is mediated by body mass index, smoking, or both (Formula: $(\log (\mathrm{OR}$ total $)-\log ($ OR_adjusted $)) / \log ($ OR_total $) \times 100))$. We replaced the values with zero for those percentage below zero. ${ }^{\text {a }}$ Total effect without any adjustment. ${ }^{b}$ Adjusted for the effect of body mass index. ${ }^{c}$ Adjusted for the effect of smoking (cigarettes per day). ${ }^{\mathrm{d}}$ Adjusted for the effects of both body mass index and smoking behaviours.

ings were consistent in sensitivity analyses and no pleiotropy was observed (Supplementary Table 5). Genetically predicted intelligence was not associated with body mass index or smoking (Supplementary Tables 4, 6 and 7).

Comparison with observational studies. The present MR findings were generally similar in the direction and magnitude to the estimates based on meta-analyses of observational studies (Supplementary Table 9). However, there were discrepancies concerning the effects of education level on suicide attempts, breast cancer and prostate cancer.

Mediation effects of body mass index and smoking. Table 2 shows the results of mediation analyses after adjusting for body mass index and smoking behaviour in the pathway from education to health outcomes. Although not apparent for all disease outcomes, body mass index and smoking partly mediated most associations between education and diseases. After adjustment for both body mass index and smoking, the direct causal effect of education on the outcomes was substantially attenuated for type 2 diabetes (64\%), major depressive disease (44\%), heart failure (36\%) and coronary artery disease (35\%).

\section{Discussion}

In the present MR study, genetic predisposition to higher education level was causally associated with the majority of major health outcomes, body mass index and smoking. Specifically, genetic predisposition to higher education level, independent of intelligence, was associated with lower risk of major depressive disorder, insomnia, suicide attempts, coronary artery disease, stroke, heart failure, breast cancer, lung cancer, type 2 diabetes, and rheumatoid arthritis. Conversely, higher education level was associated with higher risk of obsessive-compulsive disorder, bipolar disorder, anxiety, anorexia nervosa and prostate cancer. Genetically predicted higher intelligence, independent of education, was inversely related to schizophrenia. Body mass index and smoking displayed strongest mediation effects observed for type 2 diabetes, major depressive disease, heart failure and coronary artery disease.

Comparison with previous studies. Our findings are broadly in line with a vast body of observational studies showing a protective association of high educational level on major depressive disorder ${ }^{56}$, Alzheimer's disease $^{60}$, coronary heart disease $e^{61}$, heart failure ${ }^{62}$, stroke ${ }^{63}$, lung cancer ${ }^{66}$, type 2 diabetes ${ }^{67}$, chronic kidney disease $^{68}$, and obesity ${ }^{69}$. However, for suicide attempts, posttraumatic stress disorder, breast cancer and prostate cancer, our MR findings differ from observational findings. The discrepancies might be attributed by reverse causality in the observational studies, heterogeneity and small sample sizes in the meta-analyses. A substantial heterogeneity $\left(I^{2}=85 \% ; p<0.001\right)$ was observed among included observational studies in the meta-analysis of 
breast cancer ${ }^{64}$, and the sample size was small for prostate cancer ${ }^{65}$. Some studies have proposed that the higher risk of prostate cancer among men with high education level was driven by higher prostate-specific antigen screening rate among educated men compared with men with low education level ${ }^{75}$. With regard to the inverse association of higher education level with breast cancer, the association may in part be mediated by reproductive or hormone-related factors, or other health behaviours such as healthier diet and physical activity. We are not aware of any previous MR studies on education or intelligence in relation to prostate or breast cancer, but a protective causal effect of higher education on lung cancer risk has been reported recently ${ }^{16}$.

Previous MR studies showed a protective effect of higher educational level on Alzheimer's disease ${ }^{10,11}$, type 2 diabetes $^{12}$, cardiovascular disease ${ }^{13-15}$, lung cancer $^{16}$, myopia ${ }^{8}$, chronic kidney disease ${ }^{17}$ and amyotrophic lateral sclerosis $^{18}$. The present study using a larger body of SNPs as instrumental variables more precisely verified these findings and expanded the map of other health benefits of improved education level. Notably, the effects of high education level in some previous studies might be influenced by high intelligence given the tight phenotype and genetic correlation between intelligence and education level. In the present study, we used multivariable MR analysis to assess the direct effect of education level that is not mediated via intelligence. For Alzheimer's disease, we found that higher intelligence rather than education level may be the protective factor. In a previous MR study of the direct effect of education and intelligence on certain health outcomes, including diabetes, hypertension, heart attack, total stroke, total cancer, and depression, no significant association with education or intelligence was observed despite significant or suggestive associations of genetically predicted education with potential risk factors $^{20}$. Findings of other MR studies of education level in relation to obesity ${ }^{76}$ and cigarette smoking ${ }^{77}$ are $^{-}$ consistent with our findings.

Possible mechanisms. Based on results of the present MR study and previous observational studies, there are three major possible pathways linking education level to health outcomes: (1) modifiable risk factors largely mediates the educational effects on diseases ${ }^{15,78}$; (2) there may be direct effects from education-related brain structures or function change via gene methylation, gene silencing etc. ${ }^{79-81}$, especially for mental and neurological disorders; and (3) subjective well-being, happiness and meaning of life influenced by education level exerts effects on mental and somatic diseases directly or indirectly ${ }^{82-85}$. Education, as measured in this study, can be defined as an institutionalized form of social resource, and more specifically a form of cultural capital drawing on the terminology of the French sociologist Pierre Bourdieu. Related forms of cultural capital emerge as objectivized resources-such as books, art or scientific tools-or incorporated resources, such as knowledge, attitudes and practices ${ }^{86,87}$. Our study shows that education is a health relevant cultural capital whilst intelligence is not to the same degree related with health and risk of disease.

Observational studies have found that the associations between education level and diseases attenuated largely after adjustment for health-related risk factors. Compared with unadjusted model, the risk of cardiovascular diseases of low education attainment attenuated around 30-45\% in statistical models adjusted for multiple risk factors $^{88,89}$. In the present study, genetically predicted education level was associated with a favourable risk factor profile: with improved smoking behaviours as well as lower adiposity, which might mediate associations between education level and diseases. By conducting mediation analysis, we showed that body mass index and smoking behaviour partly or entirely mediated the pathway from education level to several health outcomes.

Previous studies have found that low education level might influence the changes in biochemical response and risk-related brain function, such as inflammation ${ }^{79}$, cardiometabolic traits ${ }^{80}$, and amygdala reactivity ${ }^{81}$, via gene methylation, thereby influencing disease risk. In addition, genetic studies have also revealed that improvement of subjective well-being ${ }^{82,83}$, happiness ${ }^{82,83}$, meaning of life ${ }^{84}$, social interaction ${ }^{85}$, possibly derived from high education level benefited human health directly and indirectly (e.g. influencing brain morphology, central nervous system and adrenal/pancreas tissues). There are other possible explanations, like followings: education level also could modify the risk of health outcomes through other diseases (comorbidity), the use of health care services, neighbourhood environment, occupations, income and marital status, which were amenable if education level was increased.

The results indicate that more than knowledge itself is affecting how people live their life, for instance through pathways regarding reduced smoking habits among highly educated people. Therefore, we should consider further explanations, such as the relationship between high education on the one hand and the status and resources that follow it, on the other, which could by itself have a positive health effect on the individual. A further explanation assumes that it is the process itself that can be associated with increased well-being. That is, the process of taking part of and acquiring external knowledge rather than remaining with one's own innate thinking or being kept oblivious. Should only a fraction of the disease burden be explained by this process of mental activity_-given that education leads to a different kind of thinking, which is supported by the present study in that health is affected regardless of intelligence level-then increased knowledge through education may lead to longevity through mechanisms beyond health literacy pathways of late-onset diseases and beyond the influence of social and material factors.

Strengths. The present study is the first study that comprehensively investigated the causal effects of education and intelligence on a very broad range of major disease outcomes using genetic data from large-scale GWASs and genetic consortia. We used SNPs deriving from a larger GWAS with around 1.1 million individuals as instrumental variables for education level, thereby assuring adequate statistical power to detect weak associations. In addition, we disentangled the independent effect of education level from intelligence using a multivariable MR approach. Thus, it is a straightforward approach to estimate the possible health benefits from education promotion among general population. We used mediation analysis to reveal the roles of body mass index and smoking behaviour as mediators in the pathway from education level to health outcomes. Even though there 
were genetic data for certain outcomes from GWASs with trans-ancestry populations, the majority of included participants were individuals with European ancestry thereby diminishing population stratification bias. However, population confinement limited the transferability of the present findings to populations of non-European ancestries.

Limitations. The major limitation of the present study is the possible unbalanced horizontal pleiotropy aroused from used genetic variants marking more generic biological pathways. It has been found that the lead SNPs related to education level and intelligence are significantly overexpressed in the central nervous system, such as hippocampus and cerebral cortex, but not in other organs ${ }^{26}$. For cardiovascular disease, cancers and other physical diseases, we can minimize the possibility of pleiotropy from the global or systemic measures of fitness (such as mitochondrial function). It is more likely to conclude that the potential pleiotropy might exert a large to moderate effect via predominantly neurological pathways (for example, behaviours associated with obesity or smoking) for somatic diseases. In this scenario, the vertical pleiotropy would not bias the total causal effect by a higher educational level on disease development. With regard to mental and neurological disorders, although gene overwhelmingly expressed in the brain or central nervous system, studies found no, or at most a small, genetic correlation between lower education attainment and mental and neurological disorders by using bivariate genomic-relationship-matrix restricted maximum likelihood analysis ${ }^{1}$. Thus, the associations between education level and mental or neurological diseases were not mainly because of measurable pleiotropic genetic effects, but because of education-related environmental factors. In addition, from a statistical perspective, we detected almost no pleiotropy in the results of MR-Egger regression and the estimates were consistent through sensitivity analyses, which indicated a negligible distortion by pleiotropy. Intergenerational effects from parents for certain disease, such as coronary artery diseases and type 2 diabetes, could not be assessed by using the data in the present MR study.

There was sample overlap in some analyses, which might have resulted in model overfitting if the SNPeducation associations were estimated in studies that were included in the outcome dataset. In addition, the present MR study based on summary-level data could not assess potential non-linear associations of genetically predicted education level and intelligence with outcomes. The effect size of our MR findings could not be quantitatively comparable to other studies given that we scaled the associations to one standard deviation increase in genetically predicted education and intelligence, which might differ from study to study.

In summary, the present MR study strengthened the evidence of protective role of high education level on the majority of mental disorders and somatic diseases independent of intelligence. Body mass index and smoking partly mediated several of the associations between education level and health outcomes.

\section{Data availability}

The datasets analysed in this study are publicly available summary statistics.

Received: 9 October 2020; Accepted: 8 February 2021

Published online: 22 February 2021

\section{References}

1. Peyrot, W. J. et al. The association between lower educational attainment and depression owing to shared genetic effects? Results in 25,000 subjects. Mol. Psychiatry 20, 735-743. https://doi.org/10.1038/mp.2015.50 (2015).

2. Lipnicki, D. M. et al. Age-related cognitive decline and associations with sex, education and apolipoprotein E genotype across ethnocultural groups and geographic regions: A collaborative cohort study. PLoS Med. 14, e1002261. https://doi.org/10.1371/journ al.pmed.1002261 (2017).

3. Naghavi, M. Global, regional, and national burden of suicide mortality 1990 to 2016: Systematic analysis for the Global Burden of Disease Study 2016. BMJ 364, 194. https://doi.org/10.1136/bmj.194 (2019).

4. Rosengren, A. et al. Socioeconomic status and risk of cardiovascular disease in 20 low-income, middle-income, and high-income countries: The Prospective Urban Rural Epidemiologic (PURE) study. Lancet Glob. Health 7, e748-e760. https://doi.org/10.1016/ s2214-109x(19)30045-2 (2019).

5. Albano, J. D. et al. Cancer mortality in the United States by education level and race. J. Natl. Cancer Inst. 99, 1384-1394. https:// doi.org/10.1093/jnci/djm127 (2007).

6. Putrik, P. et al. Lower education and living in countries with lower wealth are associated with higher disease activity in rheumatoid arthritis: Results from the multinational COMORA study. Ann. Rheum. Dis. 75, 540-546. https://doi.org/10.1136/annrheumdi s-2014-206737 (2016).

7. Patzer, R. E. \& McClellan, W. M. Influence of race, ethnicity and socioeconomic status on kidney disease. Nat. Rev. Nephrol. 8, 533-541. https://doi.org/10.1038/nrneph.2012.117 (2012).

8. Mountjoy, E. et al. Education and myopia: Assessing the direction of causality by mendelian randomisation. BMJ 361, k2022. https ://doi.org/10.1136/bmj.k2022 (2018).

9. Davey Smith, G. \& Ebrahim, S. What can mendelian randomisation tell us about modifiable behavioural and environmental exposures?. BMJ 330, 1076-1079. https://doi.org/10.1136/bmj.330.7499.1076 (2005).

10. Larsson, S. C. et al. Modifiable pathways in Alzheimer's disease: Mendelian randomisation analysis. BMJ 359, j5375. https://doi. org/10.1136/bmj.j5375 (2017).

11. Anderson, E. L. et al. Education, intelligence and Alzheimer's disease: Evidence from a multivariable two-sample Mendelian randomization study. Int. J. Epidemiol. 49, 1163-1172. https://doi.org/10.1093/ije/dyz280 (2020).

12. Liang, J. et al. Educational attainment protects against type 2 diabetes independently of cognitive performance: A Mendelian randomization study. Acta Diabetol. https://doi.org/10.1007/s00592-020-01647-w (2021).

13. Liao, L. Z., Zhuang, X. D., Zhang, S. Z., Liao, X. X. \& Li, W. D. Education and heart failure: New insights from the atherosclerosis risk in communities study and mendelian randomization study. Int. J. Cardiol. https://doi.org/10.1016/j.ijcard.2020.09.068 (2020).

14. Gill, D., Efstathiadou, A., Cawood, K., Tzoulaki, I. \& Dehghan, A. Education protects against coronary heart disease and stroke independently of cognitive function: Evidence from Mendelian randomization. Int. J. Epidemiol. 48, 1468-1477. https://doi. org/10.1093/ije/dyz200 (2019). 
15. Carter, A. R. et al. Understanding the consequences of education inequality on cardiovascular disease: Mendelian randomisation study. BMJ 365, 1855. https://doi.org/10.1136/bmj.11855 (2019).

16. Zhou, H. et al. Education and lung cancer: A Mendelian randomization study. Int. J. Epidemiol. 48, 743-750. https://doi.org/10.1093/ ije/dyz121 (2019).

17. Park, S. et al. Causal effects of education on chronic kidney disease: A Mendelian randomization study. Clin. Kidney J. https://doi. org/10.1093/ckj/sfaa240 (2020).

18. Zhang, L., Tang, L., Xia, K., Huang, T. \& Fan, D. Education, intelligence, and amyotrophic lateral sclerosis: A Mendelian randomization study. Ann. Clin. Transl. Neurol. 7, 1642-1647. https://doi.org/10.1002/acn3.51156 (2020).

19. van Oort, S., Beulens, J. W. J., van Ballegooijen, A. J., Burgess, S. \& Larsson, S. C. Cardiovascular risk factors and lifestyle behaviours in relation to longevity: A Mendelian randomization study. J. Intern. Med. https://doi.org/10.1111/joim.13196 (2020).

20. Davies, N. M. et al. Multivariable two-sample Mendelian randomization estimates of the effects of intelligence and education on health. Elife. https://doi.org/10.7554/eLife.43990 (2019).

21. Larsson, S. C. et al. Smoking, alcohol consumption, and cancer: A mendelian randomisation study in UK Biobank and international genetic consortia participants. PLoS Med. 17, e1003178. https://doi.org/10.1371/journal.pmed.1003178 (2020).

22. Larsson, S. C. et al. Genetic predisposition to smoking in relation to 14 cardiovascular diseases. Eur. Heart J. 41, 3304-3310. https ://doi.org/10.1093/eurheartj/ehaa193 (2020).

23. Yuan, S., Yao, H. \& Larsson, S. C. Associations of cigarette smoking with psychiatric disorders: Evidence from a two-sample Mendelian randomization study. Sci. Rep. 10, 13807. https://doi.org/10.1038/s41598-020-70458-4 (2020).

24. Larsson, S. C., Bäck, M., Rees, J. M. B., Mason, A. M. \& Burgess, S. Body mass index and body composition in relation to 14 cardiovascular conditions in UK Biobank: A Mendelian randomization study. Eur. Heart J. 41, 221-226. https://doi.org/10.1093/ eurheartj/ehz388 (2020).

25. Millard, L. A. C., Davies, N. M., Tilling, K., Gaunt, T. R. \& Davey Smith, G. Searching for the causal effects of body mass index in over 300,000 participants in UK Biobank, using Mendelian randomization. PLoS Genet. 15, e1007951. https://doi.org/10.1371/ journal.pgen.1007951 (2019).

26. Lee, J. J. et al. Gene discovery and polygenic prediction from a genome-wide association study of educational attainment in 1.1 million individuals. Nat. Genet. 50, 1112-1121. https://doi.org/10.1038/s41588-018-0147-3 (2018).

27. Savage, J. E. et al. Genome-wide association meta-analysis in 269,867 individuals identifies new genetic and functional links to intelligence. Nat. Genet. 50, 912-919. https://doi.org/10.1038/s41588-018-0152-6 (2018).

28. Stahl, E. A. et al. Genome-wide association study identifies 30 loci associated with bipolar disorder. Nat. Genet. 51, 793-803. https ://doi.org/10.1038/s41588-019-0397-8 (2019).

29. Genomic dissection of bipolar disorder and schizophrenia, including 28 subphenotypes. Cell 173, 1705-1715.e1716. https://doi. org/10.1016/j.cell.2018.05.046 (2018).

30. Howard, D. M. et al. Genome-wide meta-analysis of depression identifies 102 independent variants and highlights the importance of the prefrontal brain regions. Nat. Neurosci. 22, 343-352. https://doi.org/10.1038/s41593-018-0326-7 (2019).

31. Kunkle, B. W. et al. Genetic meta-analysis of diagnosed Alzheimer's disease identifies new risk loci and implicates Abeta, tau, immunity and lipid processing. Nat. Genet. 51, 414-430. https://doi.org/10.1038/s41588-019-0358-2 (2019).

32. Revealing the complex genetic architecture of obsessive-compulsive disorder using meta-analysis. Mol. Psychiatry 23, 1181-1188. https://doi.org/10.1038/mp.2017.154 (2018).

33. van Rheenen, W. et al. Genome-wide association analyses identify new risk variants and the genetic architecture of amyotrophic lateral sclerosis. Nat. Genet. 48, 1043-1048. https://doi.org/10.1038/ng.3622 (2016).

34. Nievergelt, C. M. et al. International meta-analysis of PTSD genome-wide association studies identifies sex-and ancestry-specific genetic risk loci. Nat. Commun. 10, 4558. https://doi.org/10.1038/s41467-019-12576-w (2019).

35. Otowa, T. et al. Meta-analysis of genome-wide association studies of anxiety disorders. Mol. Psychiatry 21, 1391-1399. https://doi. org/10.1038/mp.2015.197 (2016).

36. Jansen, P. R. et al. Genome-wide analysis of insomnia in 1,331,010 individuals identifies new risk loci and functional pathways. Nat. Genet. 51, 394-403. https://doi.org/10.1038/s41588-018-0333-3 (2019).

37. Watson, H. J. et al. Genome-wide association study identifies eight risk loci and implicates metabo-psychiatric origins for anorexia nervosa. Nat. Genet. 51, 1207-1214. https://doi.org/10.1038/s41588-019-0439-2 (2019).

38. Erlangsen, A. et al. Genetics of suicide attempts in individuals with and without mental disorders: A population-based genomewide association study. Mol. Psychiatry https://doi.org/10.1038/s41380-018-0218-y (2018).

39. Nikpay, M. et al. A comprehensive 1,000 Genomes-based genome-wide association meta-analysis of coronary artery disease. Nat. Genet. 47, 1121-1130. https://doi.org/10.1038/ng.3396 (2015).

40. Malik, R. et al. Multiancestry genome-wide association study of 520,000 subjects identifies 32 loci associated with stroke and stroke subtypes. Nat. Genet. 50, 524-537. https://doi.org/10.1038/s41588-018-0058-3 (2018).

41. Aragam, K. G. et al. Phenotypic refinement of heart failure in a national biobank facilitates genetic discovery. Circulation https:// doi.org/10.1161/circulationaha.118.035774 (2018).

42. Roselli, C. et al. Multi-ethnic genome-wide association study for atrial fibrillation. Nat. Genet. 50, 1225-1233. https://doi. org/10.1038/s41588-018-0133-9 (2018).

43. Woo, D. et al. Meta-analysis of genome-wide association studies identifies 1q22 as a susceptibility locus for intracerebral hemorrhage. Am. J. Hum. Genet. 94, 511-521. https://doi.org/10.1016/j.ajhg.2014.02.012 (2014).

44. Michailidou, K. et al. Association analysis identifies 65 new breast cancer risk loci. Nature 551, 92-94. https://doi.org/10.1038/ nature24284 (2017).

45. Schumacher, F. R. et al. Association analyses of more than 140,000 men identify 63 new prostate cancer susceptibility loci. Nat. Genet. 50, 928-936. https://doi.org/10.1038/s41588-018-0142-8 (2018).

46. Wang, Y. et al. Rare variants of large effect in BRCA2 and CHEK2 affect risk of lung cancer. Nat. Genet. 46, 736-741. https://doi. org/10.1038/ng.3002 (2014).

47. Mahajan, A. et al. Fine-mapping type 2 diabetes loci to single-variant resolution using high-density imputation and islet-specific epigenome maps. Nat. Genet. 50, 1505-1513. https://doi.org/10.1038/s41588-018-0241-6 (2018).

48. Tin, A. et al. Target genes, variants, tissues and transcriptional pathways influencing human serum urate levels. Nat. Genet. https ://doi.org/10.1038/s41588-019-0504-x (2019).

49. Okada, Y. et al. Genetics of rheumatoid arthritis contributes to biology and drug discovery. Nature 506, 376-381. https://doi. org/10.1038/nature12873 (2014).

50. de Lange, K. M. et al. Genome-wide association study implicates immune activation of multiple integrin genes in inflammatory bowel disease. Nat. Genet. 49, 256-261. https://doi.org/10.1038/ng.3760 (2017).

51. Morris, J. A. et al. An atlas of genetic influences on osteoporosis in humans and mice. Nat. Genet. 51, 258-266. https://doi. org/10.1038/s41588-018-0302-x (2019).

52. Wuttke, M. et al. A catalog of genetic loci associated with kidney function from analyses of a million individuals. Nat. Genet. 51, 957-972. https://doi.org/10.1038/s41588-019-0407-x (2019).

53. Paternoster, L. et al. Multi-ancestry genome-wide association study of 21,000 cases and 95,000 controls identifies new risk loci for atopic dermatitis. Nat. Genet. 47, 1449-1456. https://doi.org/10.1038/ng.3424 (2015). 
54. Pulit, S. L. et al. Meta-analysis of genome-wide association studies for body fat distribution in 694,649 individuals of European ancestry. Hum. Mol. Genet. 28, 166-174. https://doi.org/10.1093/hmg/ddy327 (2019).

55. Liu, M. et al. Association studies of up to 1.2 million individuals yield new insights into the genetic etiology of tobacco and alcohol use. Nat. Genet. 51, 237-244. https://doi.org/10.1038/s41588-018-0307-5 (2019).

56. Chang-Quan, H., Zheng-Rong, W., Yong-Hong, L., Yi-Zhou, X. \& Qing-Xiu, L. Education and risk for late life depression: A metaanalysis of published literature. Int. J. Psychiatry Med. 40, 109-124. https://doi.org/10.2190/PM.40.1.i (2010).

57. Li, Y. \& Cao, J. Factors associated with suicidal behaviors in mainland China: A meta-analysis. BMC Public Health 12, 524. https ://doi.org/10.1186/1471-2458-12-524 (2012).

58. Tang, B., Deng, Q., Glik, D., Dong, J. \& Zhang, L. A meta-analysis of risk factors for post-traumatic stress disorder (PTSD) in adults and children after earthquakes. Int. J. Environ. Res. Public Health. https://doi.org/10.3390/ijerph14121537 (2017).

59. Wang, M. D., Little, J., Gomes, J., Cashman, N. R. \& Krewski, D. Identification of risk factors associated with onset and progression of amyotrophic lateral sclerosis using systematic review and meta-analysis. Neurotoxicology 61, 101-130. https://doi.org/10.1016/j. neuro.2016.06.015 (2017).

60. Xu, W. et al. Meta-analysis of modifiable risk factors for Alzheimer's disease. J. Neurol. Neurosurg. Psychiatry 86, 1299-1306. https ://doi.org/10.1136/jnnp-2015-310548 (2015).

61. Khaing, W., Vallibhakara, S. A., Attia, J., McEvoy, M. \& Thakkinstian, A. Effects of education and income on cardiovascular outcomes: A systematic review and meta-analysis. Eur. J. Prev. Cardiol. 24, 1032-1042. https://doi.org/10.1177/2047487317705916 (2017).

62. Potter, E. L., Hopper, I., Sen, J., Salim, A. \& Marwick, T. H. Impact of socioeconomic status on incident heart failure and left ventricular dysfunction: Systematic review and meta-analysis. Eur. Heart J. Qual. Care Clin. Outcomes 5, 169-179. https://doi. org/10.1093/ehjqcco/qcy047 (2019).

63. McHutchison, C. A., Backhouse, E. V., Cvoro, V., Shenkin, S. D. \& Wardlaw, J. M. Education, socioeconomic status, and intelligence in childhood and stroke risk in later life: A meta-analysis. Epidemiology 28, 608-618. https://doi.org/10.1097/ede.000000000000067 5 (2017).

64. Dong, J. Y. \& Qin, L. Q. Education level and breast cancer incidence: A meta-analysis of cohort studies. Menopause https://doi. org/10.1097/gme.0000000000001425 (2019).

65. Brown, C. R. et al. Social determinants of prostate cancer in the Caribbean: A systematic review and meta-analysis. BMC Public Health 18, 900. https://doi.org/10.1186/s12889-018-5696-y (2018).

66. Sidorchuk, A. et al. Socioeconomic differences in lung cancer incidence: A systematic review and meta-analysis. Cancer Causes Control 20, 459-471. https://doi.org/10.1007/s10552-009-9300-8 (2009).

67. Bellou, V., Belbasis, L., Tzoulaki, I. \& Evangelou, E. Risk factors for type 2 diabetes mellitus: An exposure-wide umbrella review of meta-analyses. PLoS ONE 13, e0194127. https://doi.org/10.1371/journal.pone.0194127 (2018).

68. Zeng, X. et al. Associations between socioeconomic status and chronic kidney disease: A meta-analysis. J. Epidemiol. Community Health 72, 270-279. https://doi.org/10.1136/jech-2017-209815 (2018).

69. He, J., Chen, X., Fan, X., Cai, Z. \& Huang, F. Is there a relationship between body mass index and academic achievement? A metaanalysis. Public Health 167, 111-124. https://doi.org/10.1016/j.puhe.2018.11.002 (2019).

70. Bowden, J., Davey Smith, G., Haycock, P. C. \& Burgess, S. Consistent estimation in mendelian randomization with some invalid instruments using a weighted median estimator. Genet. Epidemiol. 40, 304-314. https://doi.org/10.1002/gepi.21965 (2016).

71. Burgess, S. \& Thompson, S. G. Interpreting findings from Mendelian randomization using the MR-Egger method. Eur. J. Epidemiol. 32, 377-389. https://doi.org/10.1007/s10654-017-0255-x (2017).

72. Rees, J. M. B., Wood, A. M. \& Burgess, S. Extending the MR-Egger method for multivariable Mendelian randomization to correct for both measured and unmeasured pleiotropy. Stat. Med. 36, 4705-4718. https://doi.org/10.1002/sim.7492 (2017).

73. Spiller, W., Davies, N. M. \& Palmer, T. M. Software Application Profile: mrrobust-a tool for performing two-sample summary Mendelian randomization analyses. Int. J. Epidemiol. https://doi.org/10.1101/142125 (2017).

74. Hemani, G. et al. The MR-Base platform supports systematic causal inference across the human phenome. Elife. https://doi. org/10.7554/eLife.34408 (2018).

75. Lund Nilsen, T. I., Johnsen, R. \& Vatten, L. J. Socio-economic and lifestyle factors associated with the risk of prostate cancer. $B r$. J. Cancer 82, 1358-1363. https://doi.org/10.1054/bjoc.1999.1105 (2000).

76. Bockerman, P. et al. Does higher education protect against obesity? Evidence using Mendelian randomization. Prev. Med. 101, 195-198. https://doi.org/10.1016/j.ypmed.2017.06.015 (2017).

77. Gage, S. H., Bowden, J., Davey Smith, G. \& Munafo, M. R. Investigating causality in associations between education and smoking: A two-sample Mendelian randomization study. Int. J. Epidemiol. 47, 1131-1140. https://doi.org/10.1093/ije/dyy131 (2018).

78. Zeng, L. et al. Genetically modulated educational attainment and coronary disease risk. Eur. Heart J. 40, 2413-2420. https://doi. org/10.1093/eurheartj/ehz328 (2019).

79. Stringhini, S. et al. Life-course socioeconomic status and DNA methylation of genes regulating inflammation. Int. J. Epidemiol. 44, 1320-1330. https://doi.org/10.1093/ije/dyv060 (2015).

80. Huang, J. Y. et al. Accounting for life-course exposures in epigenetic biomarker association studies: Early life socioeconomic position, candidate gene DNA methylation, and adult cardiometabolic risk. Am. J. Epidemiol. 184, 520-531. https://doi.org/10.1093/ aje/kww014 (2016).

81. Swartz, J. R., Hariri, A. R. \& Williamson, D. E. An epigenetic mechanism links socioeconomic status to changes in depressionrelated brain function in high-risk adolescents. Mol. Psychiatry 22, 209-214. https://doi.org/10.1038/mp.2016.82 (2017).

82. Song, L. et al. Polygenic score of subjective well-being is associated with the brain morphology in superior temporal gyrus and insula. Neuroscience 414, 210-218. https://doi.org/10.1016/j.neuroscience.2019.05.055 (2019).

83. Okbay, A. et al. Genetic variants associated with subjective well-being, depressive symptoms, and neuroticism identified through genome-wide analyses. Nat. Genet. 48, 624-633. https://doi.org/10.1038/ng.3552 (2016).

84. Baselmans, B. M. L. \& Bartels, M. A genetic perspective on the relationship between eudaemonic-and hedonic well-being. Sci. Rep. 8, 14610. https://doi.org/10.1038/s41598-018-32638-1 (2018).

85. Day, F. R., Ong, K. K. \& Perry, J. R. B. Elucidating the genetic basis of social interaction and isolation. Nat. Commun. 9, 2457. https ://doi.org/10.1038/s41467-018-04930-1 (2018).

86. Richardson, J. G. Handbook of Theory and Research for the Sociology of Education. (Greenwood Press, 1986).

87. Bourdieu, P. Distinction: A Social Critique of the Judgement of Taste. (Routledge, 2010).

88. Manrique-Garcia, E., Sidorchuk, A., Hallqvist, J. \& Moradi, T. Socioeconomic position and incidence of acute myocardial infarction: A meta-analysis. J. Epidemiol. Community Health 65, 301-309. https://doi.org/10.1136/jech.2009.104075 (2011).

89. Stringhini, S. et al. Association of socioeconomic position with health behaviors and mortality. JAMA 303, 1159-1166. https:// doi.org/10.1001/jama.2010.297 (2010).

\section{Acknowledgements}

Summary-level data for education level, intelligence and outcomes were obtained from the UK Biobank and genetic consortia, including Psychiatric Genomics Consortium (bipolar disorder, schizophrenia, obsessive-compulsive disorder, post-traumatic stress disorder, anxiety and anorexia nervosa), The International Genomics 
of Alzheimer's Project (IGAP), Project MinE, Center for Neurogenomics and Cognitive Research (CNCR), iPSYCH, CARDIoGRAMplusC4D Consortium, MEGASTROKE Consortium, Atrial Fibrillation Consortium (AFGen), International Stroke Genetics Conosrtium (ISGC), Breast Cancer Association Consortium (BCAC), The Prostate Cancer Association Group to Investigate Cancer Associated Alterations in the Genome (PRACTICAL) consortium, The International Lung Cancer Consortium (ILCCO), The DIAbetes Genetics Replication And Meta-analysis (DIAGRAM) consortium, The Global Urate Genetics Consortium (GUGC), Genetics and Allied research in Rheumatic diseases Networking (GARNET) consortium, UK Inflammatory Bowel Disease Genetics (IBD) Consortium, CKDGen Consortium, the EArly Genetics and Lifecourse Epidemiology (EAGLE) Consortium, GEnetic Factors for OSteoporosis (GEFOS) Consortium, GIANT consortium, Consortium of Alcohol and Nicotine use (GSCAN). The authors thank all investigators for sharing these data. The list of investigators of MEGASTROKE is available at http://megastroke.org/authors.html. Funding of the MEGASTROKE project is specified at megastroke.org/acknowledgements.html.

\title{
Author contributions
}

S.Y. designed the study, conducted the systematic review, performed the statistical analyses, drafted the manuscript, contributed to the interpretation of the results and critical revision of the manuscript for important intellectual content and approved the final version of the manuscript. Y.X. conducted the systematic review, contributed to the interpretation of the results and critical revision of the manuscript for important intellectual content and approved the final version of the manuscript. M.M. contributed to the interpretation of the results and critical revision of the manuscript for important intellectual content and approved the final version of the manuscript. K.M. contributed to the interpretation of the results and critical revision of the manuscript for important intellectual content and approved the final version of the manuscript. S.C.L. designed the study, contributed to the interpretation of the results and critical revision of the manuscript for important intellectual content and approved the final version of the manuscript.

\section{Funding}

Open access funding provided by Uppsala University. Funding for this study came from the Swedish Research Council (Vetenskapsrådet; Grant Number 2019-00977), the Swedish Research Council for Health, Working Life and Welfare (Forte; Grant Number 2018-00123), and the Swedish Heart-Lung Foundation (Hjärt-Lungfonden; Grant number 20190247).

\section{Competing interests}

All authors declared no support from any organization for the submitted work; no financial relationships with any organization that might have an interest in the submitted work in the previous three years; no other relationships or activities that could appear to have influenced the submitted work.

\section{Additional information}

Supplementary Information The online version contains supplementary material available at https://doi. org/10.1038/s41598-021-83801-0.

Correspondence and requests for materials should be addressed to S.C.L.

Reprints and permissions information is available at www.nature.com/reprints.

Publisher's note Springer Nature remains neutral with regard to jurisdictional claims in published maps and institutional affiliations.

\begin{abstract}
Open Access This article is licensed under a Creative Commons Attribution 4.0 International (c) License, which permits use, sharing, adaptation, distribution and reproduction in any medium or
format, as long as you give appropriate credit to the original author(s) and the source, provide a link to the Creative Commons licence, and indicate if changes were made. The images or other third party material in this article are included in the article's Creative Commons licence, unless indicated otherwise in a credit line to the material. If material is not included in the article's Creative Commons licence and your intended use is not permitted by statutory regulation or exceeds the permitted use, you will need to obtain permission directly from the copyright holder. To view a copy of this licence, visit http://creativecommons.org/licenses/by/4.0/.
\end{abstract}

(C) The Author(s) 2021 